\title{
On the singular homology of one class of simply-connected cell-like spaces
}

\author{
Katsuya Eda, Umed H. Karimov and Dušan Repovš
}

\begin{abstract}
In our earlier papers we constructed examples of 2-dimensional nonaspherical simply-connected cell-like Peano continua, called Snake space. In the sequel we introduced the functor $S C(-,-)$ defined on the category of all spaces with base points and continuous mappings. For the circle $S^{1}$, the space $S C\left(S^{1}, *\right)$ is a Snake space. In the present paper we study the higher-dimensional homology and homotopy properties of the spaces $S C(Z, *)$ for any path-connected compact spaces $Z$.
\end{abstract}

Mathematics Subject Classification (2010). Primary: 54G15, 54G20, 54F15; Secondary: 54F35, 55Q52.

Keywords. Snake space, Topologist sine curve, asphericity, simple connectivity, cell-likeness, semi-local strong contractibility, continuum, free $\sigma$-product of groups, van Kampen theorem.

\section{Introduction}

It is well-known that there exist planar noncontractible continua $X$ all homotopy groups $\pi_{i}(X), i \geq 1$, of which are trivial (e.g. the Warsaw circle). Every planar simply connected Peano continum is a contractible space, see e.g. 10, 14. Noncontractible homology locally connected (HLC and therefore Peano) continua, all homotopy groups of which are trivial, were constructed in 9]. All these examples are infinite-dimensional. The following problem remains open [6]:

Problem 1.1. Does there exist a finite-dimensional noncontractible Peano continuum all homotopy groups of which are trivial?

We constructed in [4] the functor $S C(-,-)$, defined on the category of all topological spaces with base points. Roughly speaking, for any space $Z$, one takes the infinite cylinder $Z \times[0, \infty)$ and attaches it to the square $[0,1] \times[-1,1] \subset \mathbb{R}^{2}$, along the open Topologist sine curve:

$$
\left\{(x, y) \in \mathbb{R}^{2} \mid y=\sin (1 / x), 0<x \leq 1\right\},
$$


so that its diameter tends to zero. The space $S C(Z, *)$ is called the Snake cone and when $Z$ is the circle $S^{1}$, the space $S C\left(S^{1}, *\right)$ is called the Snake space.

The Snake space was the first candidate for an example of a simply connected aspherical noncontractible Peano continuum. However, we have discovered, rather unexpectedly, that the group $\pi_{2}\left(S C\left(S^{1}\right)\right)$ is nontrivial [6].

It is easy to see that the Snake space is a cell-like Peano continuum (for the verification of cell-likeness use e.g. [12]). We have already proved the following:

Theorem 1.2. 4, Theorem 1.1] For every path-connected space Z, the Snake cone $S C(Z)$ is simply-connected.

Our original proof in 4] was quite long and technical. We shall give a short proof of this result in Section 2 of the present paper.

We proved in [6] that whenever $\pi_{1}\left(Z, z_{0}\right)$ is nontrivial, the singular homology group $H_{2}(S C(Z) ; \mathbb{Z})$ is nontrivial, and since the spaces $S C(Z)$ are simply connected, it follows by the Hurewicz Theorem, that $\pi_{2}\left(S C(Z), z_{0}\right)$ is isomorphic to $H_{2}(S C(Z) ; \mathbb{Z})$, and hence is also nontrivial. The converse was proved in [7, along the lines of the proof in [4. In Section 3 of the present paper we shall give a different and significantly shorter proof of the generalized result for $(n-1)$-connected spaces, when $n \geq 2$.

Theorem 1.3. ([7, Theorem 1.1] for $n=2$.) Let $Z$ be any $(n-1)$-connected space, $n \geq 2$. Then $H_{n}(S C(Z) ; \mathbb{Z})$ and $\pi_{n}\left(S C(Z), z_{0}\right)$ are trivial.

Since by [6, Theorem 3.1] the nontriviality of $\pi_{1}(Z)$ implies that of $H_{2}(S C(Z))$, we obtain the following:

Corollary 1.4. For any path-connected space $Z$ and any point $z_{0} \in Z$, the following statements are equivalent:

(i) $\pi_{1}\left(Z, z_{0}\right)$ is trivial;

(ii) $\mathrm{H}_{2}(S C(Z) ; \mathbb{Z})$ is trivial; and

(iii) $\pi_{2}\left(S C(Z), z_{0}\right)$ is trivial.

Undefined notions are the usual ones and we refer the reader to [15].

\section{Proof of Theorem 1.2}

We shall follow the notations for the Snake cone $S C(Z)$ and the projection $p: S C(Z) \rightarrow \mathbb{I}^{2}$ as in [4]. The polygonal line $A_{1} B_{1} A_{2} B_{2} \cdots$ on $\mathbb{I}^{2}$, together with the limit interval $A B$, is the piecewise linear version of the Topologist sine curve in Figure 1. 


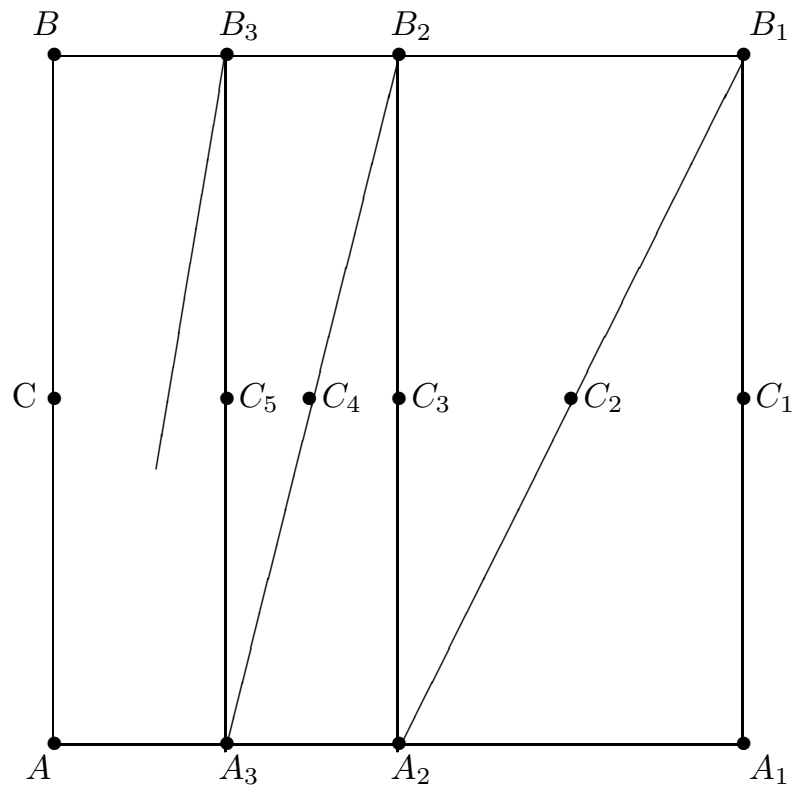

Figure 1

For a proof of Theorem 1.2, we recall the notion of the free $\sigma$-product of groups and a lemma from [2]. An element of $\mathbb{x}_{i \in I}^{\sigma} G_{i}$ is expressed by a word $W \in \mathcal{W}^{\sigma}\left(G_{i}: i \in I\right)$, where $G_{i} \cap G_{j}=\{e\}$ for $i \neq j, W: \bar{W} \rightarrow \bigcup\left\{G_{i}: i \in I\right\}$, $\bar{W}$ is a countable linearly ordered set, and $W^{-1}\left(G_{i}\right)$ is finite for each $i \in I$ (cf. [2]). Let $h: \mathbb{X}_{i \in I}^{\sigma} G_{i} \rightarrow \mathbb{X}_{j \in J}^{\sigma} H_{j}$ be a homomorphism.

For any $W \in \mathcal{W}^{\sigma}\left(G_{i}: i \in I\right)$, express $h(W(\alpha))$ by a reduced word $V_{\alpha} \in \mathcal{W}^{\sigma}\left(H_{j}: j \in J\right)$. Define $\bar{V}$ to be $\left\{(\alpha, \beta): \alpha \in \bar{W}, \beta \in \overline{V_{\alpha}}\right\}$ with the lexicographical ordering and $V(\alpha, \beta)=V_{\alpha}(\beta)$. A homomorphism $h$ is said to be standard, if $V$ as defined above, is a word in $\mathcal{W}^{\sigma}\left(H_{j}: j \in J\right)$ and $h(W)=V$, for every $W \in \mathcal{W}^{\sigma}\left(G_{i}: i \in I\right)$. We have used the superscript ${ }^{\sigma}$ in some cases, which means a restriction to the countable case. Hence, when an index set is countable, the restriction is unnecessary and we drop the superscript $^{\sigma}$.

Let $\left\{\left(X_{i}, x_{i}\right)\right\}_{i \in I}$ be pointed spaces. Let $\left(\widetilde{\bigvee}_{i \in I}\left(X_{i}, x_{i}\right), x^{*}\right)$ be a bouquet of $\left\{\left(X_{i}, x_{i}\right)\right\}_{i \in I}$. The underlying set $\left(\widetilde{V}_{i \in I}\left(X_{i}, x_{i}\right), x^{*}\right)$ is the quotient space of a discrete union of all $X_{i}$ 's by the identification of all points $x_{i}$ with a singleton $x^{*}$ and the topology is defined by specifying the neighborhood bases as follows (c.f. [1):

(1) If $x \in X_{i} \backslash\left\{x_{i}\right\}$, then the neighborhood base of $x$ in $\widetilde{\bigvee}_{i \in I}\left(X_{i}, x_{i}\right)$ is the one of $X_{i}$;

(2) The point $x^{*}$ has a neighborhood base, each element of which is of the form:

$$
\widetilde{\bigvee}_{i \in I \backslash F}\left(X_{i}, x_{i}\right) \vee \bigvee_{j \in F} U_{j}
$$


where $F$ is a finite subset of $I$ and each $U_{j}$ is an open neighborhood of $x_{j}$ in $X_{j}$ for $j \in F$.

Lemma 2.1. [2, Theorem A.1] Suppose that the space $X_{i}$ is locally simplyconnected and first countable at $x_{i}$, for each $i \in I$. Then

$$
\pi_{1}\left(\widetilde{\bigvee_{i \in I}}\left(X_{i}, x_{i}\right), x^{*}\right) \simeq \mathbb{x}_{i \in I}^{\sigma} \pi_{1}\left(X_{i}, x_{i}\right)
$$

Lemma 2.2. 3, Proposition 2.10] Let $X_{i}$ and $Y_{j}$ be locally simply-connected and first countable at $x_{i}$ and $y_{j}$, respectively for each $i \in I$ and $j \in J$. Then for the continuous map

$$
\left.f:\left(\widetilde{\bigvee}_{i \in I}\left(X_{i}, x_{i}\right), x^{*}\right) \rightarrow \widetilde{\left(\widetilde{\bigvee}_{j \in J}\right.}\left(Y_{j}, y_{j}\right), y^{*}\right)
$$

the induced homomorphism

$$
f_{*}: \pi_{1}\left(\widetilde{\bigvee}_{i \in I}\left(X_{i}, x_{i}\right), x^{*}\right) \rightarrow \pi_{1}\left(\widetilde{\bigvee}_{j \in J}\left(Y_{j}, y_{j}\right), y^{*}\right)
$$

is standard under the natural identifications:

$$
\begin{aligned}
& \pi_{1}\left(\widetilde{\bigvee}_{i \in I}\left(X_{i}, x_{i}\right), x^{*}\right)=\mathbb{x}_{i \in I}^{\sigma} \pi_{1}\left(X_{i}, x_{i}\right) \text { and } \\
& \pi_{1}\left(\widetilde{\bigvee}_{j \in J}\left(Y_{j}, y_{j}\right), y^{*}\right)=\mathbb{x}_{j \in J}^{\sigma} \pi_{1}\left(Y_{j}, y_{j}\right) .
\end{aligned}
$$

Let $Y_{0}=p^{-1}(\mathbb{I} \times[0,2 / 3))$ and $Y_{1}=p^{-1}(\mathbb{I} \times(1 / 3,1])$. Then $S C(Z)=$ $Y_{0} \cup Y_{1}$ and $Y_{0} \cap Y_{1}$ is open in $S C(Z)$. We let $i_{0}: Y_{0} \cap Y_{1} \rightarrow Y_{0}, i_{1}: Y_{0} \cap Y_{1} \rightarrow Y_{1}$, $j_{0}: Y_{0} \rightarrow S C(X), j_{1}: Y_{1} \rightarrow S C(X)$, and $i: Y_{0} \cap Y_{1} \rightarrow S C(X)$ be the inclusion maps.

Proof of Theorem 1.2. We observe that $p^{-1}(\mathbb{I} \times\{1 / 2\})$ is a strong deformation retract of $Y_{0} \cap Y_{1}$. Let $C_{n}$ be the points on $\mathbb{I} \times\{1 / 2\}$ such that $C_{2 n-1}$ is on the segment $A_{n} B_{n}$ and $C_{2 n}$ is on the segment $B_{n} A_{n+1}$. Let $X_{n}$ be the subspace $\left[C, C_{n}\right] \cup p^{-1}\left(\left\{C_{n}\right\}\right)$ of $S C(Z)$. Then $Y_{0} \cap Y_{1}$ is homotopy equivalent to $\widetilde{V}_{n<\omega}\left(X_{n}, C\right)$. Since $X_{n}$ is locally simply connected and first countable at $C$ and $p^{-1}\left(\left\{C_{n}\right\}\right)$ is homeomorphic to $Z, \pi_{1}\left(Y_{0} \cap Y_{1}\right)$ is isomorphic to $\mathbb{x}_{n<\omega} \pi_{1}\left(p^{-1}\left(\left\{C_{n}\right\}\right) \cong \mathbb{x}_{n<\omega} \pi_{1}(Z)\right.$ by Lemmas 2.1 and 2.2. Simlilarly, $\pi_{1}\left(Y_{0}\right)$ and $\pi_{1}\left(Y_{1}\right)$ are isomorphic to $\mathbb{x}_{n<\omega} \pi_{1}\left(p^{-1}\left(\left\{A_{n}\right\}\right) \cong \mathbb{x}_{n<\omega} \pi_{1}(Z)\right.$ and $\mathbb{x}_{n<\omega} \pi_{1}\left(p^{-1}\left(\left\{B_{n}\right\}\right) \cong \mathbb{x}_{n<\omega} \pi_{1}(Z)\right.$ respectively. Here we remark that $i_{0 *}$ and $i_{1 *}$ are standard homomorphisms under these presentations of the fundamental groups.

Since $Y_{0}, Y_{1}$ and $Y_{0} \cap Y_{1}$ are path-connected and open in $S C(Z)$, we can apply the van Kampen theorem [13, Theorem 2.1] for homomorphisms $i_{0 *}, i_{1 *}, j_{0 *}, j_{1 *}$ and $i_{*}$ between fundamental groups. The diagram formed by these five homomorphisms is a pushout diagram and hence the ranges of $j_{0 *}$ and $j_{1 *}$ generate $\pi_{1}(S C(Z))$. Therefore $i_{*}$ is surjective. For the simple connectivity of $S C(Z)$ it suffices to show that $i_{*}$ is trivial.

We let $u_{n} \in \pi_{1}\left(p^{-1}\left(\left\{C_{n}\right\}\right)\right.$ be the copy of $u \in \pi_{1}(Z)$. Let $U_{n}$ be the word

$$
u_{n}^{-1} u_{n+1} \cdots u_{n+2 k}^{-1} u_{n+2 k+1} \cdots .
$$


Since $i_{0 *}\left(u_{2 m}^{-1} u_{2 m+1}\right)=e$ and $i_{1 *}\left(u_{2 m-1}^{-1} u_{2 m}\right)=e$ and $i_{0 *}$ and $i_{1 *}$ are standard homomorphisms, $i_{0 *}\left(U_{2 m}\right)=e, i_{1 *}\left(U_{2 m}\right)=i_{1 *}\left(u_{2 m}^{-1}\right), i_{0 *}\left(U_{2 m-1}\right)=$ $i_{0 *}\left(u_{2 m-1}^{-1}\right)$, and $i_{1 *}\left(U_{2 m-1}\right)=e$.

Now, an arbitrary element of $\pi_{1}\left(Y_{0} \cap Y_{1}\right)$ is expressed by a word

$$
W \in \mathcal{W}\left(\pi_{1}\left(p^{-1}\left(\left\{C_{n}\right\}\right)\right): n \in \mathbb{N}\right) .
$$

For each letter $u_{n} \in \pi_{1}\left(p^{-1}\left(\left\{C_{n}\right\}\right)\right)$ for an odd $n$ appearing in $W$, we insert $U_{n+1}$ successively to $u_{n}$ and form $W^{*}$. Since

$$
U_{n+1} \in \mathcal{W}\left(\pi_{1}\left(p^{-1}\left(\left\{C_{m}\right\}\right)\right): m \geq n\right),
$$

$W^{*}$ is actually a word in $\mathcal{W}\left(\pi_{1}\left(p^{-1}\left(\left\{C_{n}\right\}\right)\right): n \in \mathbb{N}\right)$. We let $W_{0}$ be the word obtained by deleting all letters in $\bigcup \pi_{1}\left(p^{-1}\left(\left\{C_{2 n-1}\right\}\right)\right)$ from $W$. Since $i_{0 *}$ and $i_{1 *}$ are standard homomorphisms, $i_{0 *}\left(W^{*}\right)=i_{0 *}(W)$ and $i_{1 *}\left(W^{*}\right)=i_{1 *}\left(W_{0}\right)$. Now

$$
W_{0} \in \mathcal{W}\left(\pi_{1}\left(p^{-1}\left(\left\{C_{2 n}\right\}\right)\right): n \in \mathbb{N}\right) .
$$

We again insert $U_{n+1}$ for each letter $u_{n}$ appearing in $W_{0}$ and form $W_{0}^{*}$. Then, by the symmetrical argument as above, we conclude that $i_{1 *}\left(W_{0}^{*}\right)=i_{1 *}\left(W_{0}\right)$ and $i_{0 *}\left(W_{0}^{*}\right)=e$. Now,

$$
\begin{aligned}
i_{*}(W) & =j_{0 *} \circ i_{0 *}\left(W^{*}\right)=j_{1 *} \circ i_{1 *}\left(W_{0}\right) \\
& =j_{1 *} \circ i_{1 *}\left(W_{0}^{*}\right)=j_{0 *} \circ i_{0 *}\left(W_{0}^{*}\right)=j_{0 *}(e)=e,
\end{aligned}
$$

which imples that $\pi_{1}(S C(Z))$ is indeed trivial.

\section{Proof of Theorem 1.3}

For every group $G_{i}, \Pi_{i \in I}^{\sigma} G_{i}$ is the subgroup of $\prod_{i \in I} G_{i}$ consisting of elements $u$ such that $\{i \in I: u(i) \neq 0)\}$ is countable. A space $X$ is called semi-locally strongly contractible at $x \in X$, if there exists an open neighborhood $U \subset X$ of $x$ such that there exists a contraction of $U$ in $X$ to $x$ which fixes $x$ (cf. [8]).

Lemma 3.1. 8, Theorem 1.1] Let $n \geq 2$ and let $X_{i}$ be a space which is $(n-1)$ connected semi-locally strongly contractible at $x_{i}$ for each $i \in I$. Then

$$
\pi_{n}\left(\widetilde{\bigvee}_{i \in I}\left(X_{i}, x_{i}\right), x^{*}\right) \cong \Pi_{i \in I}^{\sigma} \pi_{n}\left(X_{i}, x_{i}\right)
$$

Proof of Theorem 1.3. We shall use the Mayer-Vietoris sequence instead of the van Kampen theorem (as in the preceding proof). Consider the following Mayer-Vietoris homology exact sequence (over $\mathbb{Z})$ for the triad $\left(S C(Z) ; Y_{0}, Y_{1}\right)$ from Section 2:

$H_{n}\left(Y_{0} \cap Y_{1}\right) \stackrel{i_{0 *}+i_{1 *}}{\longrightarrow} H_{n}\left(Y_{0}\right) \oplus H_{n}\left(Y_{1} \stackrel{j_{0 *}+j_{1 *}}{\longrightarrow} H_{n}(S C(Z)) \stackrel{\partial}{\longrightarrow} H_{n-1}\left(Y_{0} \cap Y_{1}\right)\right.$.

Since $Z$ is $(n-1)$-connected, $Y_{0} \cap Y_{1}$ is also $(n-1)$-connected, which implies that $H_{n-1}\left(Y_{0} \cap Y_{1}\right)=\{0\}$. Therefore it suffices to show that $i_{0 *}+i_{1 *}$ is surjective.

Note that $Y_{0} \cap Y_{1}, Y_{0}$ and $Y_{1}$ are simply connected and that $p^{-1}(\mathbb{I} \times$ $\{1 / 2\}), p^{-1}(\mathbb{I} \times\{0\})$ and $p^{-1}(\mathbb{I} \times\{1\})$ are strong deformation retracts of $Y_{0} \cap Y_{1}, Y_{0}$ and $Y_{1}$, respectively. For the same reason as explained in the 
first paragraph of the proof of Theorem 1.2, the local properties required in Lemma 3.1 for $Y_{0} \cap Y_{1}, Y_{0}$ and $Y_{1}$ are satisfied and we have:

$$
\begin{aligned}
H_{n}\left(Y_{0} \cap Y_{1}\right) & =\pi_{n}\left(Y_{0} \cap Y_{1}\right)=\Pi_{m=1}^{\infty} H_{n}\left(p^{-1}\left(\left\{C_{m}\right\}\right)\right), \\
H_{n}\left(Y_{0}\right) & =\pi_{n}\left(Y_{0}\right)=\prod_{m=1}^{\infty} H_{n}\left(p^{-1}\left(\left\{A_{m}\right\}\right)\right), \text { and } \\
H_{n}\left(Y_{1}\right) & =\pi_{n}\left(Y_{1}\right)=\Pi_{m=1}^{\infty} H_{n}\left(p^{-1}\left(\left\{B_{m}\right\}\right)\right),
\end{aligned}
$$

where $A_{m}, B_{m}, C_{m}$ are the points indicated in Figure 1. Since $p^{-1}\left(\left\{C_{m}\right\}\right)$, $p^{-1}\left(\left\{A_{m}\right\}\right)$ and $p^{-1}\left(\left\{B_{m}\right\}\right)$ are homeomorphic to $Z$, we can identify the homology groups of these spaces with $H_{n}(Z)$. Therefore for $u \in \Pi_{m=1}^{\infty} H_{n}\left(p^{-1}\left(\left\{C_{m}\right\}\right)\right)$

$$
\begin{aligned}
i_{0 *}(u)(1) & =u(1), \\
i_{0 *}(u)(m) & =u(2 m-1)+u(2 m-2) \quad \text { for } m \geq 2, \\
i_{1 *}(u)(m) & =u(2 m-1)+u(2 m) \quad \text { for } m \geq 1 .
\end{aligned}
$$

For any given $v \in H_{n}\left(Y_{0}\right), w \in H_{n}\left(Y_{1}\right)$, define:

$$
\begin{aligned}
u(2 m-1) & =\Sigma_{k=1}^{m} v(k)-\Sigma_{k=1}^{m-1} w(k) \text { and } \\
u(2 m) & =\Sigma_{k=1}^{m} w(k)-\Sigma_{k=1}^{m} v(k) .
\end{aligned}
$$

Then $i_{0 *}(u)=v$ and $i_{1 *}(u)=w$ and hence $\left(i_{0 *}+i_{1 *}\right)(u)=v+w$. We have thus shown that $H_{2}(S C(Z), \mathbb{Z})$ is trivial and consequently $\pi_{2}(S C(Z))$ is also trivial by the Hurewicz Theorem and Theorem 1.2

Remark 3.2. The proof of Theorem [1.3] in [7] was along the same line as the proof of [4, Theorem 1.1], which contains a procedure to avoid $p^{-1}((0,1] \times$ $\{1\})$. The use of the Mayer-Vietoris sequence above makes it possible for us to skip this procedure, as does the use of the van Kampen theorem in the proof of Theorem 1.2 . When $Z$ is not simply-connected, we cannot avoid $p^{-1}((0,1] \times\{1\})$ for $\pi_{2}(S C(Z))$, which reflects the nontriviality of $\pi_{2}(S C(Z))$ in Theorem 1.3 .

We remark that the presentations of the homotopy groups, i.e. Lemmas 2.1, 2.2 and 3.1 are also useful to make proofs shorter. That is, our previous proofs implicitly contain the procedures used in the proofs of the Mayer-Vietoris sequence, the van Kampen theorem and the lemmas.

\section{Acknowledgements}

The authors thank the referee for several comments and suggestions. This research was supported by the Slovenian Research Agency grants P1-02920101, J1-9643-0101 and J1-2057-0101. The first author was also supported by the Grant-in-Aid for Scientific research (C) of Japan No. 20540097.

\section{References}

[1] M. G. Barrat and J. Milnor, An example of anomalous singular homology, Proc. Amer. Math. Soc. 13 (1962), 293-297. 
[2] K. Eda, Free $\sigma$-products and noncommutatively slender groups, J. Algebra 148 (1992), 243-263.

[3] - The fundamental groups of one-dimensional wild spaces and the Hawaiian earring, Proc. Amer. Math. Soc. 130 (2002), 1515-1522.

[4] K. Eda, U. Karimov, and D. Repovš, A construction of simply connected noncontractible cell-like two-dimensional Peano continua, Fund. Math. 195 (2007), 193-203.

[5] _ On the fundamental group of $\mathbf{R}^{3}$ modulo the Case-Chamberin continuum, Glasnik Mat. 42 (2007), 89-94.

[6] _ A nonaspherical cell-like 2-dimensional simply connected continuum and related constructions, Topology Appl. 156 (2009), 515-521.

[7] - The second homotopy group of $S C(Z)$, Glasnik Mat. 44(64) (2009), 493-498.

[8] K. Eda and K. Kawamura, Homotopy groups and homology groups of the $n$ dimensional Hawaiian earring, Fund. Math. 165 (2000), 17-28.

[9] U. Karimov and D. Repovš On noncontractible compacta with trivial homology and homotopy groups, Proc. Amer. Math. Soc. 138:4 (2010), 1525-1531.

[10] U. Karimov, D. Repovš, W. Rosicki and A. Zastrow On two dimensional planar compacta not homotopy equivalent to any one-dimensional compactum, Topol. Appl. 153 (2005), 284-293.

[11] K. Kuratowski, Topology II, Academic Press, New York, 1968.

[12] S. Mardešić and J. Segal, Shape theory, North-Holland, Amsterdam, 1982

[13] W. Massey, Algebraic Topology: An Introduction, Grad. Texts Math. 56, Springer, Berlin 1984.

[14] S. B. Nadler, Jr., Continuum Theory. An Introduction, Monogr. and Textbooks in Pure and Appl. Math. 158, Marcel Dekker, Inc., New York, 1992.

[15] E. H. Spanier, Algebraic Topology, McGraw-Hill, New York, 1966.

Katsuya Eda

School of Science and Engineering, Waseda University, Tokyo 169-8555, Japan

e-mail: eda@logic.info.waseda.ac.jp

Umed H. Karimov

Institute of Mathematics, Academy of Sciences of Tajikistan, Ul. Ainy $299^{A}$, Dushanbe 734063, Tajikistan

e-mail: umedkarimov@gmail.com

Dušan Repovš

Faculty of Mathematics and Physics, and Faculty of Education, University of Ljubljana, P.O.Box 2964, Ljubljana 1001, Slovenia

e-mail: dusan.repovs@guest.arnes.si 